RESEARCH ARTICLE

\title{
Prime-boost vaccination with plasmid DNA and a chimeric adenovirus type 5 vector with type 35 fiber induces protective immunity against HIV
}

\author{
K-Q Xin ${ }^{1}$, N Jounai ${ }^{1}$, K Someya ${ }^{2}$, K Honma ${ }^{3}$, H Mizuguchi ${ }^{4}$, S Naganawa ${ }^{5}$, K Kitamura ${ }^{5}$,

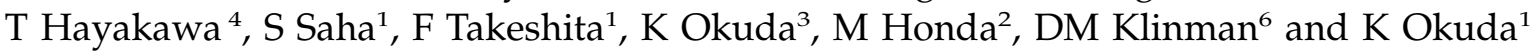 \\ ${ }^{1}$ Department of Molecular Biodefense Research, Yokohama City University, Graduate School of Medicine, Yokohama, Japan; ${ }^{2}$ AIDS \\ Research Center, National Institute of Infectious Diseases, Tokyo, Japan; ${ }^{3}$ Department of Microbiology, Tokyo Dental College, Chiba, \\ Japan; ${ }^{4}$ Laboratory of Gene Transfer and Regulation National Institute of Biomedical Innovation, Osaka, Japan; ${ }^{5}$ Department of Public \\ Health, Yokohama City University, Graduate School of Medicine, Yokohama, Japan; and ${ }^{6}$ Center for Biologics Evaluation and Research, \\ US Food and Drug Administration, Bethesda, MD, USA
}

\begin{abstract}
Immunization involving a DNA vaccine prime followed by an adenovirus type 5 (Ad5) boost elicited a protective immune response against SHIV challenge in monkeys. However, the hepatocellular tropism of Ad5 limits the safety of this viral vector. This study examines the safety and immunogenicity of a replication-defective chimeric Ad5 vector with the Ad35 fiber $(A d 5 / 35)$ in $B A L B / C$ mice and rhesus monkeys. This novel Ad5/35 vector showed minimal hepatotoxicity after intramuscular administration with the novel Ad5/35 vector. In addition, an Ad5/35 vector expressing HIV Env gp160 protein
\end{abstract}

(Ad5/35-HIV) generated strong HIV-specific immune responses in both animal models. Priming with a DNA vaccine followed by Ad5/35-HIV boosting yielded protection against a gp160-expressing vaccinia virus challenge in $B A L B / c$ mice. The Ad5/35-HIV vector was significantly less susceptible to the pre-existing Ad5 immunity than a comparable Ad5 vector. These findings indicate that an Ad5/35 vector-based HIV vaccine may be of considerable value for clinical use. Gene Therapy (2005) 12, 1769-1777. doi:10.1038/ sj.gt.3302590; published online 4 August 2005

Keywords: Ad5/35 vector; HIV; animal model; vaccine; immune response

\section{Introduction}

A vaccine capable of preventing HIV infection is needed to control the global AIDS pandemic. In the past decade, multiple strategies to produce an immunogenic HIV vaccine have been explored. This included production of HIV subunit peptide vaccines, ${ }^{1}$ DNA vaccines, ${ }^{2}$ recombinant virus-vector vaccines (including modified vaccinia virus, $^{3}$ adenovirus (Ad) ${ }^{4,5}$ rabies virus, ${ }^{6}$ flavivirus, ${ }^{7}$ sendai virus, ${ }^{8}$ Venezuelan equine encephalitis virus, ${ }^{9}$ and adeno-associated virus ${ }^{10,11}$ ), and bacterial vectorvaccines (bacille Calmette-Guerin, ${ }^{12,13}$ and Lactococcus lactis $\left.{ }^{14}\right)$. Each of these strategies showed some promising results in animal models, either alone or in combination.

Among these vectors, the replication-defective human Ad type 5 (Ad5) recombinants (with the deletion of a replication-essential gene, E1) and the replication-defective modified vaccinia Ankara (MVA) elicited the most potent $\mathrm{CD}^{+} \mathrm{T}$-cell responses and provided the highest degree of protection in non-human primates. ${ }^{3,4,15,16} \mathrm{~A}$ major limitation for the clinical application of the Ad5 and MVA vectors is the pre-existing immunity against

Correspondence: Dr K Okuda, Department of Molecular Biodefense Research, Yokohama City University, Graduate School of Medicine, 3-9 Fukuura, Kanazawa-ku, Yokohama 236-0004, Japan

Received 3 April 2005; accepted 18 June 2005; published online 4 August 2005 these viruses in humans, since most of the human population has been infected with $\mathrm{Ad} 5^{17}$ and vaccinia virus on being administered the smallpox vaccine. The preexisting antiviral immunity may strongly influence the efficacy of the HIV vaccine using Ad5 and MVA vectors.

Human Ads are classified into six subgroups from A-F. ${ }^{18}$ Most of Ad serotypes belonging to subgroups A, $C, D, E$, and $F$ use the coxsackievirus and adenovirus receptor (CAR) as a cellular receptor. ${ }^{19}$ The Ad5 (subgroup C) has well-defined biological properties and has been widely used as a vector for gene therapy and vaccine. The replication-defective Ad5 vector can easily be produced in high titers and is highly effective in boosting HIV-specific immunity. ${ }^{4,15}$ However, this virus uses CAR as its primary attachment receptor, which confers tropism for liver parenchymal cells. ${ }^{19-22}$ This raises important safety concerns, ${ }^{22}$ particularly because the administration of an Ad5-based vector for gene therapy resulted in the death of a patient. ${ }^{23}$ In response to these shortcomings, our laboratory has examined the immunogenicity and safety of a replication-defective chimeric Ad5 vector with Ad type 35 fiber (Ad5/35) (Ad35 virus was classified as subgroup B). The Ad35 fiber showed $25 \%$ amino-acid homology with the Ad5 fiber. ${ }^{24}$ Cell entry of Ad35 is CAR independent and may involve CD46 receptor, which expresses on most human cells. ${ }^{25}$ Ad35 can be transducted to liver nonparenchymal cells on a level 4-5 log orders lower than Ad5, but not to 
liver parenchymal cells. ${ }^{20}$ In the present study, we found that the Ad5/35 recombinants not only induced strong antigen-specific humoral and cellular immune responses and exhibited minimal hepatotoxicity in both mice and non-human primates, but were also significantly less susceptible to the pre-existing Ad5 immunity than a comparable Ad5 vector.

\section{Results}

\section{Biodistribution of Ad in mice}

In the initial experiments, mice were injected intramuscularly (i.m.) with $10^{11}$ viral particles (vp) of a luciferaseexpressing Ad5 (Ad5-Luc) or Ad5/35 vector (Ad5/ 35-Luc). Luciferase expression was monitored using an in vivo imaging system (IVIS) on days 3 and 10 after administration. As shown in Figure 1a, all of the Ad5/ $35-L u c$ vector remained at the injection site. In contrast, substantial amounts of the Ad5 vector migrated to the liver. This difference in vector distribution was confirmed by studies involving LacZ-expressing Ad5 and Ad5/35 vectors (data not shown). Studies on serum glutamic-oxaloacetic transaminase (GOT) and serum glutamic-pyruvic transaminase (GPT) levels revealed that mice injected with the Ad5-Luc vector had changes indicative of liver damage (Figure 1b). We also analyzed serum levels of key proinflammatory cytokines (IFN- $\gamma$ and IL-6) on days 0,3 , and 10 after administration of virus vectors. The levels of IFN- $\gamma$ and IL-6 were significantly elevated following administration of Ad5Luc vector, but not of Ad5/35-Luc vector (Figure 1c). Thus, the hepatotoxicity caused by the Ad5 vector was circumvented by the use of an Ad5/35 vector.

Time-course study of HIV-specific immune responses in mice. Ad5/35 vector can efficiently transfect antigen-presenting cells $s^{18,21,26,27}$ and muscular cells (Figure 1a). In order to explore whether the virus vector can be used as a vaccine vector, we constructed an HIV Env gp160-expressing Ad5/35 vector (Ad5/35-HIV). The expression of HIV gp160 was confirmed by Western blotting (Figure 2a). The HIV Env gp160-expressing DNA vaccine (DNA-HIV) used in this study was reported previously. ${ }^{28}$ The mice were immunized with $10^{10} \mathrm{vp}$ of Ad5/35-HIV vector, and the HIV-specific cellular immune response was periodically monitored by the intracellular cytokine staining (ICS) assay. The assay has been widely utilized to distinguish the relative contributions of $\mathrm{CD}^{+}$cells to the overall T-cell responses. ${ }^{29}$ On day 3, HIV-specific IFN- $\gamma$-secreting $\mathrm{CD}^{+} \mathrm{T}$ cells can be detected (Figure $2 \mathrm{~b}$ ) and peaked 2 weeks after immunization. On day 50 and month 7 after final immunization, 2.5 and $1.2 \%$ of HIVspecific IFN- $\gamma$-secreting $\mathrm{CD}^{+} \mathrm{T}$ cells still persisted, respectively.

Mice were vaccinated with Ad5/35-HIV vector to explore the humoral immune response 7 weeks after the final immunization. The animals immunized with $10^{10} \mathrm{vp}$ of Ad5/35-HIV vector developed a high-tittered anti-gp160 antibody (Ab) response (Figure 2c). The specificity of the $\mathrm{Ab}$ response was confirmed by Western blotting (Figure 2c, upper panel). The magnitude of this response was not significantly altered by preimmunization with the DNA-HIV vaccine (Figure 2c). DNA-HIV a
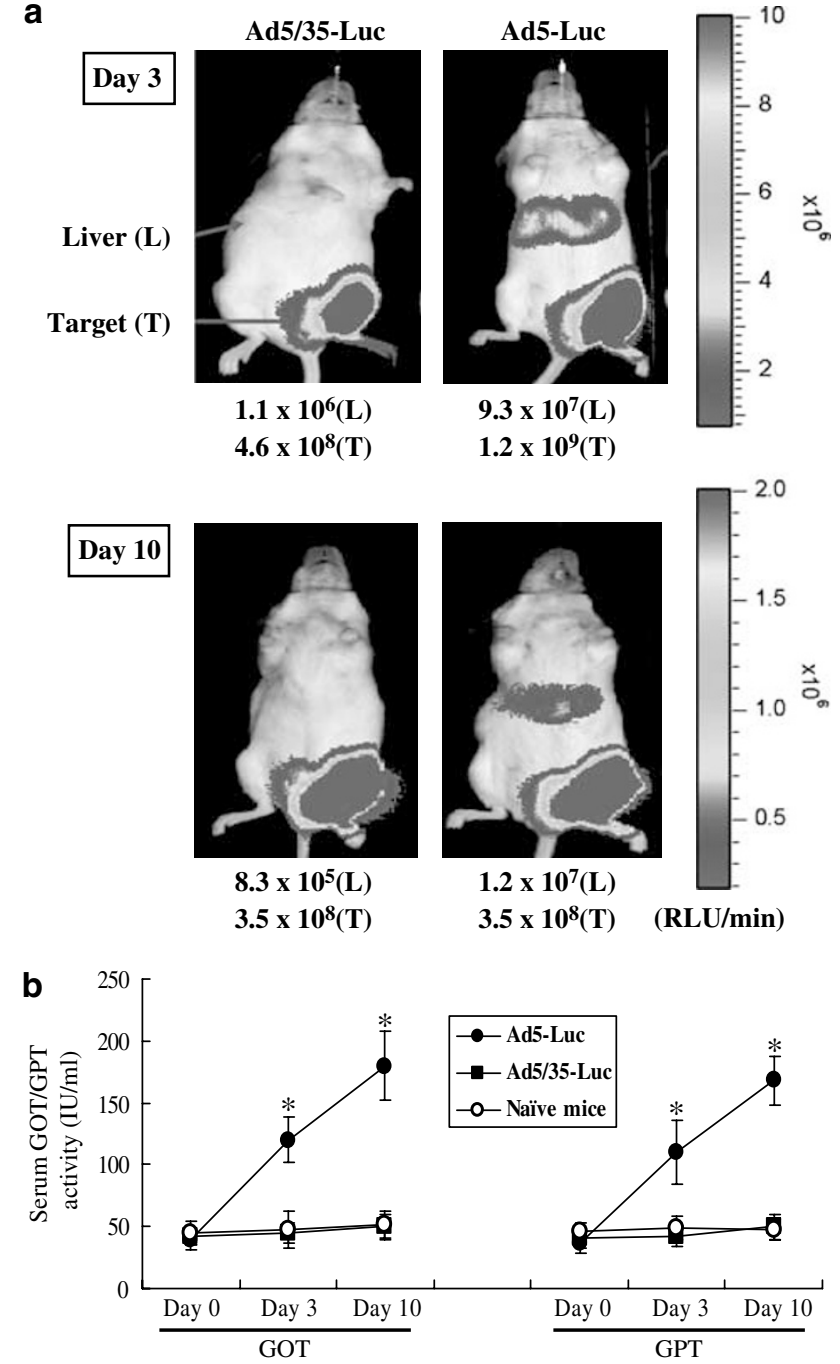

C

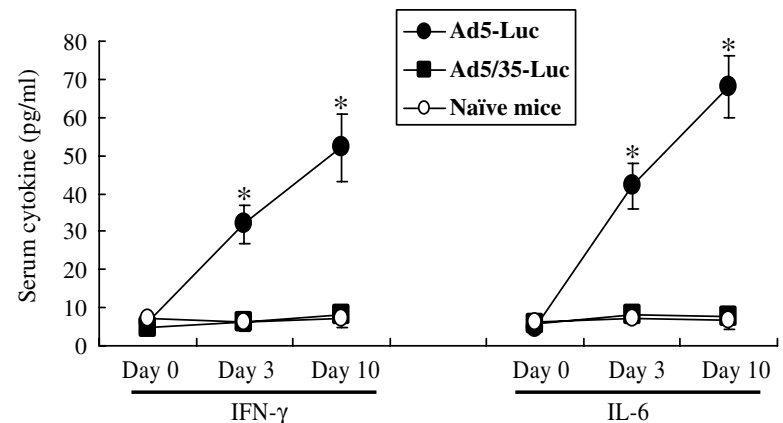

Figure 1 Biodistribution and safety of Ad vectors. BALB/c mice were injected i.m. with $10^{11}$ vp of the Ad5-Luc or Ad5/35-Luc vector. (a) Using an IVIS CCD camera, vector distribution was detected after the addition of luciferin (3 mice/group) (expressed in relative light units (RLU)). One of the mice is represented and other mice used show the same pattern. (b) Serum GOP and GPT levels were measured on days 0, 3, and 10 after injection (5 mice/group). IU: international unit. (c) Serum IFN- $\gamma$ and IL-6 levels were measured on days 0,3 , and 10 after injection (5 mice/group). *Mean values are significantly different between Ad5-Luc-administered mice and Ad5/35-Luc-administered mice or naïve mice at the same time point.

vaccination alone generated a low level of HIV-specific serum $\mathrm{Ab}$ (Figure 2c, bottom panel). HIV-specific neutralizing $\mathrm{Ab}$ was only detectable in the Ad5/35-HIV 
a 293 cells DNA-HIV Ad5/35-HIV

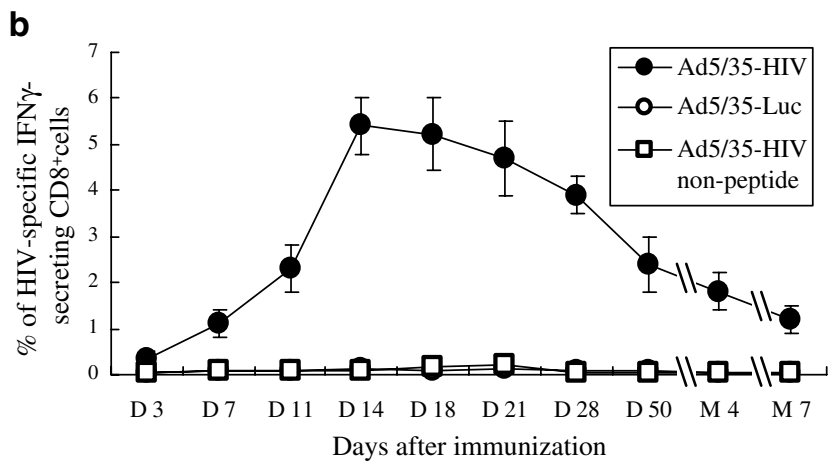

C
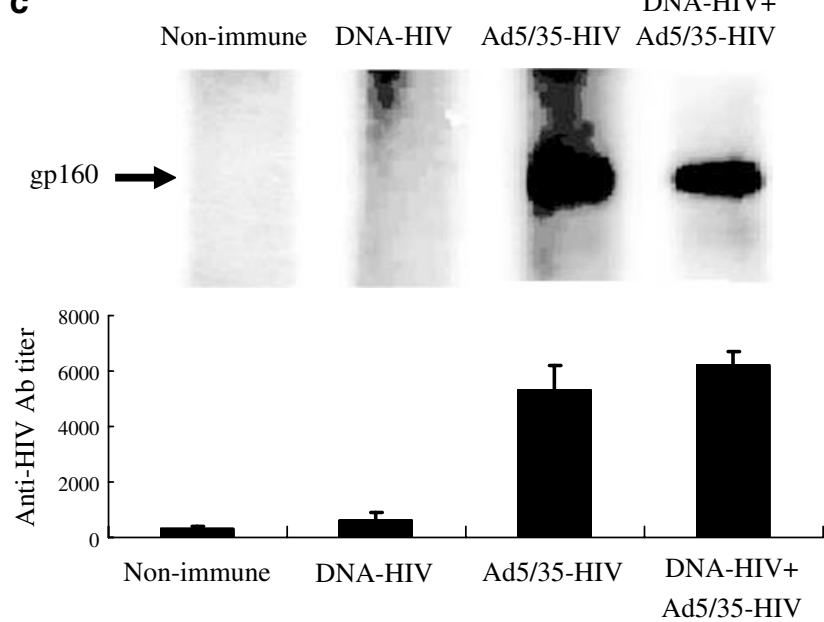

Figure 2 Time course of HIV-specific immune responses in mice. (a) HIV Env protein expression of DNA-HIV vaccine and Ad5/35-HIV on HEK293 cells was confirmed by Western blotting using an HIV Envspecific $m A b$. (b) Time-course study of cellular immune responses after a single i.m. injection of $10^{10}$ op of Ad5/35-HIV vector (3 mice/time point). $D$ : day; M: month. (c) HIV-specific Ab was detected by Western blotting using 100-fold diluted antisera (serum pool of 10 mice/group) (upper panel) and ELISA (10 mice/group) (bottom left panel).

vaccinated mice (1:186) and DNA prime/Ad5/35-HIV boosted mice (1:206).

Immune responses and challenge in mice 2 weeks after vaccination. There is growing evidence that cellular immunity contributes to protecting the host against HIV infection. . $^{3,430,31}$ The ability of the Ad5/35 vector to trigger the activation and proliferation of antigen-specific $\mathrm{T}$ cells was monitored. Vaccination with the DNA-HIV vaccine induced the number of HIV- specific IFN- $\gamma$-secreting $\mathrm{CD} 8^{+} \mathrm{T}$ cells to increase from background levels $(<0.2-0.7 \%)(P<0.05)$ (Table 1$)$. This was significantly less than the effect of vaccination with the Ad5/35-HIV vector $\left(10^{10} \mathrm{vp} /\right.$ mouse $)$ that increased the IFN- $\gamma$-secreting $\mathrm{CD}^{+} \mathrm{T}$ cells to $5.5 \% \quad(P<0.05)$. Priming with the DNA-HIV vaccine followed by an Ad5/35-HIV vector boost led to a further three-fold increase in the number of IFN- $\gamma$-secreting $\mathrm{CD}^{+} \mathrm{T}$ cells $(P<0.05)$.

A tetramer-binding assay was used to verify that the IFN- $\gamma$-secreting cells were MHC class I-restricted HIVspecific $\mathrm{CD}^{+}$T cells. ${ }^{32}$ A single immunization with Ad5/ 35-HIV vector elicited a significant increase in the number of tetramer-binding CD8 ${ }^{+}$T cells (Table 1 ). When compared with DNA-HIV vaccination alone, immunization with the Ad5/35-HIV vector yielded five-fold more HIV-specific $\mathrm{CD}^{+} \mathrm{T}$ cells $(P<0.05)$. Priming with the DNA-HIV vaccine, followed by Ad5/35-HIV boosting, further increased the tetramer binding $(P<0.05)$.

To examine the protective activity of the Ad5/35-HIV vector, immunized mice were challenged with $10^{8}$ plaque forming units (PFU) of vPE16 2 weeks after final immunization. The animals that were vaccinated with the Ad5/35 vector alone or in combination with the DNA-HIV vaccine were completely protected from infection (Table 1); however, the DNA-HIV vaccination alone had little impact on the susceptibility to infection by vPE16.

Long-term cell-medicated immune responses and challenge in mice. The durability of these vaccine regimens was explored. HIV-specific cellular immune responses persisted through 7 months after final immunization (Table 1 and Figure 2b). To determine whether this immune response was protective, vaccinated mice were challenged with vPE16 $\left(10^{8} \mathrm{PFU} /\right.$ mouse) 7 weeks after final immunization. The viral load of Ad5/35-HIV-immunized mice was reduced by $10^{5}$ as compared with that of the control mice $(P<0.05)$. DNAHIV vaccination by itself was not protective, but the combination of DNA-HIV priming and Ad5/35-HIV boosting yielded a prolonged and complete protection (Table 1).

\section{Biodistribution of $A d$ in rhesus macaques}

To study the biodistribution of Ad in monkeys, $10^{11} \mathrm{vp}$ of Ad5-Luc and Ad5/35-Luc vectors was injected i.m. into two rhesus monkeys for each vector. The luciferase activity in the tissues was detected 3 days after administration, because high luciferase activity in the mouse liver was observed at that time point. Liver infection with Ad5 vector was 20- to 40-fold stronger than that with Ad5/35 vector (Figure 3a). It is important to note that the luciferase activity of the cerebellum and the posterior cerebrum in the monkeys that received the Ad5-Luc vector was two- and four-fold higher, respectively, than that of the monkeys that received the Ad5/ 35-Luc vector.

Immune response in rhesus monkeys after vaccination To explore the immunogenicity of the Ad5/35-HIV vector in monkeys, two rhesus macaques were immunized i.m. with $10^{11} \mathrm{vp}$ of Ad5/35-HIV vector. A detectable HIV-specific serum $\mathrm{Ab}$ response developed 
Table 1 HIV-specific cell-mediated immune responses and virus challenge after vaccination

\begin{tabular}{|c|c|c|c|c|c|c|c|c|}
\hline & \multicolumn{3}{|c|}{ Week 2} & \multicolumn{3}{|c|}{ Week 7} & \multirow{2}{*}{$\begin{array}{c}\text { Month } 4 \\
\text { Tetramer (\%) }\end{array}$} & \multirow{2}{*}{$\begin{array}{c}\text { Month } 7 \\
\text { Tetramer (\%) }\end{array}$} \\
\hline & $\operatorname{ICS}(\%)$ & Tetramer (\%) & Ovary viral titer & $\operatorname{ICS}(\%)$ & Tetramer (\%) & Ovary viral titer & & \\
\hline Nonimmune & $0.1 \pm 0.1$ & $0.1 \pm 0.1$ & $8 \times 10^{8} \pm 35$ & $0.1 \pm 0.1$ & $0.1 \pm 0.1$ & $1 \times 10^{9} \pm 65$ & $0.0 \pm 0.0$ & $0.0 \pm 0.0$ \\
\hline DNA-Empty & $0.1 \pm 0.1$ & $0.1 \pm 0.1$ & $2 \times 10^{9} \pm 45$ & $0.0 \pm 0.0$ & $0.0 \pm 0.0$ & $8 \times 10^{8} \pm 32$ & $0.0 \pm 0.0$ & $0.0 \pm 0.0$ \\
\hline Ad5/35-Luc & $0.2 \pm 0.1$ & $0.2 \pm 0.2$ & $2 \times 10^{9} \pm 25$ & $0.0 \pm 0.0$ & $0.0 \pm 0.0$ & $4 \times 10^{8} \pm 46$ & $0.0 \pm 0.0$ & $0.0 \pm 0.0$ \\
\hline DNA-HIV & $0.7 \pm 0.1$ & $1.0 \pm 0.3$ & $6 \times 10^{6} \pm 42$ & $0.4 \pm 0.2$ & $0.6 \pm 0.1$ & $5 \times 10^{7} \pm 51$ & $0.3 \pm 0.1$ & $0.1 \pm 0.1$ \\
\hline Ad5/35-HIV & $5.5 \pm 0.3$ & $5.2 \pm 0.3$ & $\mathrm{ND}^{-}$ & $2.5 \pm 0.8$ & $3.1 \pm 0.2$ & $2 \times 10^{3} \pm 34$ & $2.5 \pm 0.5$ & $1.2 \pm 0.4$ \\
\hline DNA-HIV+Ad5/35-HIV & $17.2 \pm 0.8$ & $19.4 \pm 2.1$ & ND & $8.2 \pm 1.2$ & $8.9 \pm 0.8$ & ND & $7.1 \pm 0.6$ & $4.1 \pm 0.3$ \\
\hline
\end{tabular}

Mice were immunized with DNA plasmid or Ad5/35 vector, either alone or in combination. At 2 weeks, 7 weeks, 4 months, and 7 months after final immunization, HIV-specific cellular immune responses were detected by ICS assay and tetramer assay. The data represent the percentage of IFN- $\gamma$ - or tetramer-positive CD8 ${ }^{+}$T cells (5-10 mice/group). The backgrounds were less than $0.1 \%$ IFN- $\gamma$-secreting CD8 ${ }^{+}$T cells when cells were stimulated with control peptide (influenza NP peptide, TYQRTRALV). The vaccinated mice (10 mice/group) were challenged with vaccinia virus vPE16 2 or 7 weeks after final immunization. At 6 days after the challenge, the vPE16 titer in mouse ovaries was measured. ND, not detectable.
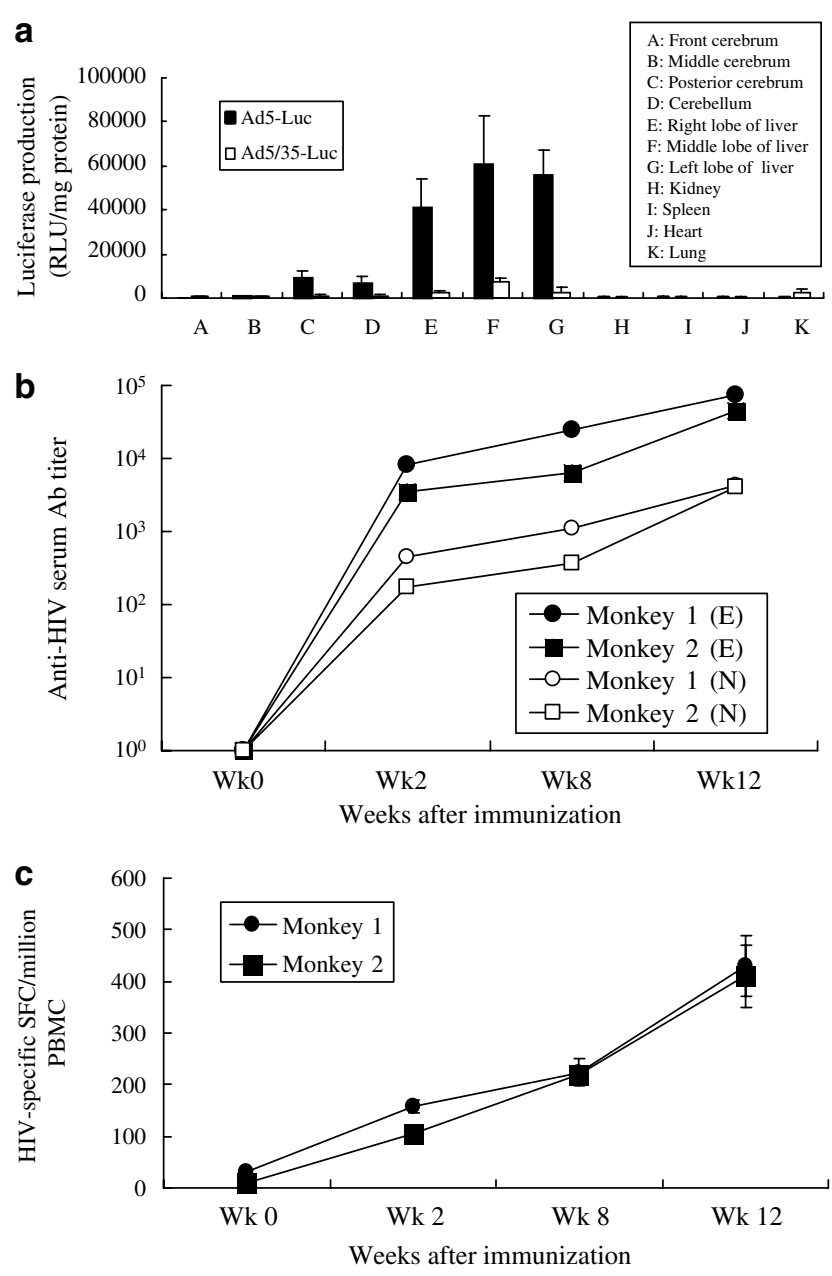

Figure 3 Biodistribution and HIV-specific immune responses in rhesus monkeys. Rhesus monkeys (2 monkeys/group) were administered i.m. $10^{11}$ vp of Ad5-Luc or Ad5/35-Luc vector. The luciferase activity in the organs of the monkey (expressed in RLU) was examined 3 days after administration (a). Rhesus monkeys were immunized i.m. with $10^{11}$ op of Ad5/35-HIV vector at 0 and 8 weeks. PBMCs were isolated at weeks 0,2 , 8, and 12. HIV-specific Ab titers were measured in triplicate by ELISA (E) $(\bullet, \mathbf{0})$ and neutralizing assay $(N)(O, \square)(b)$. The detecting limitation of the neutralizing assay was $100 \mathrm{ND}_{50} / \mathrm{ml}$. PBMCs were stimulated with HIV Env gp120 protein, and the number of cells activated to secrete IFN- $\gamma$ was determined in triple wells by ELISPOT (c). SFC: spot-forming cells. within 2 weeks of immunization (Figure 3b). The animals were boosted at 8 weeks. After 4 weeks, titers in excess of 1:50 000 were achieved. Similar results were observed in neutralizing $\mathrm{Ab}$. A increase in the number of HIVspecific IFN- $\gamma$-secreting T cells was also detected in the peripheral blood mononuclear cells (PBMCs) (Figure 3c). Boosting with Ad5/35-HIV vector further increased this T-cell response.

\section{Effect of pre-existing immunity on vaccination}

To evaluate the effect of the anti-Ad5 neutralization $\mathrm{Ab}$ (found in $60 \%$ of the adult human population) ${ }^{17}$ on the Ad5/35 vector, the infectivity of the vector was examined after incubation with serially diluted serum from subjects with high titers of anti-Ad5 Abs (anti-Ad5 neutralizing titer $=1: 64)$. As shown in Figure 4 , the human antisera had 1:8 anti-Ad5/35 neutralizing titer and normal human sera against either Ad5 or Ad5/35 vector was less than 1:4. The sera derived from Ad5/35HIV-immunized monkeys showed two-fold higher neutralizing $\mathrm{Ab}$ titer against $\mathrm{Ad} 5 / 35$ vector than $\mathrm{Ad} 5$ vector.

To examine the effect of pre-existing anti-Ad5 immunity on the activity of the Ad5/35 vector in vivo, mice were injected i.m. with $10^{10}$ or $10^{11}$ vp of Ad5-Luc. After 8 weeks, these animals were immunized with $10^{10} \mathrm{vp}$ of Ad5-HIV or Ad5/35-HIV. The HIV-specific responses were detected by the tetramer assay 2 weeks after immunization. Although pre-existing immunity to Ad5 reduced the immune response elicited by both vectors, Ad5/35-HIV was significantly more immunogenic than Ad5-HIV $(P<0.05$; Figure 4$)$.

\section{Discussion}

This study demonstrates that an Ad5/35-HIV vector vaccine induces strong cellular and humoral immune responses with minimal toxicity in mice and rhesus macaques. A prime-boost strategy involving the DNAHIV vaccine and the Ad5/35-HIV vector generated protective immunity against viral infection in mice.

A widely used HIV vaccine should have high immunogenicity, low cost of production, and low or no pathogenicity. Replication-defective Ad5 is one of the best vectors for HIV vaccine development. Vaccination 
with recombinant Ad5 has achieved great success in inducing protection against virus infection in several animal models. ${ }^{4,15,33}$ Ad5 is well characterized, and its subclinical disease association in humans is well known. ${ }^{34,35}$ However, a majority of the human population (more than 60\%) is infected with the Ad5 virus. ${ }^{17,36,37}$ The neutralizing $\mathrm{Ab}$ and the cellular immune responses against the Ad5 fiber and capsid may reduce the efficacy of the Ad5 vector when it is used in a clinical trial. ${ }^{37,38}$ The switching of the Ad serotypes ${ }^{37,39}$ and the use of animal Ads ${ }^{40-44}$ enables the partial bypass of the preexisting immune responses to Ad5 viruses. However, there are a few drawbacks: lack of knowledge regarding the biology of these viruses, including tropism on human cells; potential difficulties in manufacturing; and the possibility of in vivo recombination with other human viruses leading to unknown diseases. Animal Ad vectors may induce the antigen-specific responses as strongly as Ad5 in animal models. ${ }^{43}$ However, their immunogenicity in humans is still unknown. This study used a chimera Ad5 vector with Ad35 fiber, which relates with cell tropism. The Ad5/35, similar to Ad5, has a high productive titer in tissue culture cells, because it is commonly known that human subgroup B Ads, such as Ad5, have a considerably higher titer as compared with

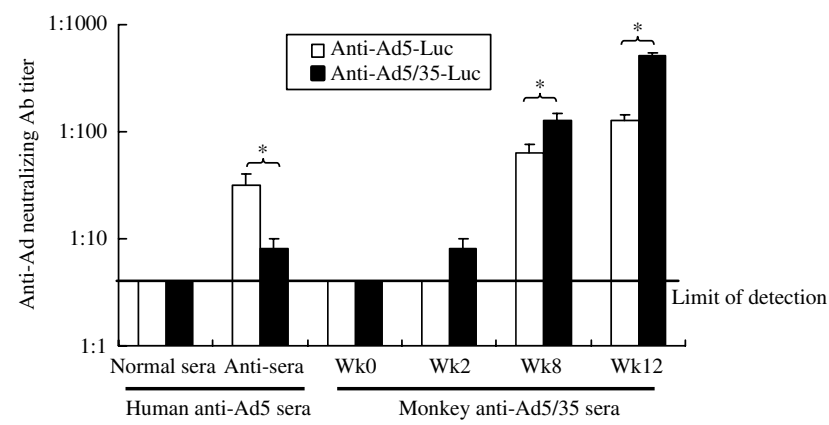

Figure 4 Effect of pre-existing antiviral immunity. Ad5-Luc and Ad5/35Luc vectors were incubated with an equal volume of serially diluted normal human sera (No. 2, anti-Ad5 neutralization titer $<1: 4$ ), human antisera (No. 2, anti-Ad5 neutralization titer $=64$ ), or monkey antisera from Figure $3 b$ and $c$ (No. 2) in triplicate and were subsequently added to infected Vero cells in a 96-well plate at $10^{7}$ vp/well. The luciferase activity was measured $48 \mathrm{~h}$ after infection. The neutralizing titer was calculated with limited serum dilution when the luciferase activity in the Ad-infected cells was equal with the background. Average and standard deviations for three independent experiments are shown. ${ }^{*}$ Mean values are significantly different between groups. other subgroup viruses, including Ad35. Nevertheless, the virus displayed the cell tropism of Ad35. We explored the immunogenicity of the Ad5/35 vector encoding HIV Env gene in both mice and non-human primates. The results indicate that the Ad5/35-HIV vector elicited strong HIV-specific humoral and cellular immune responses that conferred protective immunity (Table 1 and Figure $3 b$ and c). Coupled with the evidence that an Ad5/35 vector transduces human dendritic cells more efficiently as compared with an Ad5 vector, ${ }^{18,21,26,27}$ these findings suggest that the Ad5/35-HIV vector is a promising candidate for human trials.

Another concern regarding the use of the Ad5 vector in clinical trials is its strong tropism to hepatocytes that is caused by the high expression of CAR in the hepatocytes. Our experiments showed a high expression of the Ad5 vector in the liver in both mice and non-human primates after i.m. administration, but not of the Ad5/35 vector (Figure 1). In contrast to Ad5 vector, Ad/35 vector did not elevate the levels of serum markers (GOT/GPT) of hepatotoxicity and key proinflammatory cytokines (IFN$\gamma$ and IL-6) in mice (Figure $1 \mathrm{~b}$ and $\mathrm{c}$ ). These results demonstrate that, as a vaccine vector, $\mathrm{Ad} 5 / 35$ vector is safer than Ad5 vector. However, low expression of Ad5/ 35 vector in monkey liver was still detected after i.m. administration of Ad5/35-Luc vector to monkeys (Figure 3a). It may have resulted from low capacity of Ad5/35 to infect liver nonparenchymal cells, but not liver parencymal cells. ${ }^{20}$ Interestingly, we found a certain magnitude of Ad5 vector expression in the posterior cerebrum and cerebellum of monkeys; however, the Ad5/35 vector was not expressed (Figure 4a). Nevertheless, in the present experiment, we could not precisely define the location of the Ad5-infected cells or determine whether the infection potentially causes local inflammation or toxicity. However, potential brain infection after Ad5 vector administration is a safety concern because intranasal administration of the Ad5 vector has been reported to result in the infection of the central nervous system. ${ }^{45}$

In this study, the effect of pre-existing anti-Ad5 immunity on the Ad5/35 vector was explored along with several immunization protocols as follows. (1) Both in vitro and in vivo studies demonstrate that the Ad5/35 vector is significantly less susceptible to neutralization by anti-Ad5 Abs as compared with a conventional Ad5 vector (Table 2 and Figure 4). The administration or infection of Ad can induce immune responses against the Ad hexon, penton, and fiber antigens. The exchange of fiber can partially reduce the inhibition of the

Table 2 Effect of pre-existing antiviral immunity

\begin{tabular}{|c|c|c|c|c|}
\hline & Prime & Anti-Ad5 neutralizing Ab titer & Boost & Tetramer assay (\%) \\
\hline Control & Non & $<1: 4$ & $\begin{array}{l}\text { Ad5-HIV (10 } 10 \mathrm{vp} / \text { mouse }) \\
\text { Ad5/35-HIV (10 }\end{array}$ & $\begin{array}{l}4.8 \pm 0.2 \\
51+02\end{array}$ \\
\hline Low dose & Ad5-Luc $\left(10^{10} \mathrm{vp} /\right.$ mouse $)$ & 1:102 & $\begin{array}{r}\left.\text { Ad5-HIV (10 } 10^{10} \mathrm{vp} / \text { mouse }\right) \\
\left.\text { Ad5/35-HIV (10 } 10^{10} \mathrm{vp} / \text { mouse }\right)\end{array}$ & $\left.\begin{array}{l}2.3 \pm 0.4 \\
4.6 \pm 0.6\end{array}\right\} *$ \\
\hline High dose & Ad5-Luc (10 $10^{11} \mathrm{vp} /$ mouse $)$ & $1: 248$ & $\begin{array}{r}\left.\text { Ad5-HIV (10 } 10^{10} \mathrm{vp} / \text { mouse }\right) \\
\text { Ad5/35-HIV (10 } \mathrm{vp} / \text { mouse })\end{array}$ & $\left.\begin{array}{l}0.5 \pm 0.1 \\
2.6 \pm 0.4\end{array}\right\} *$ \\
\hline
\end{tabular}

After 8 weeks, naive mice or mice pretreated with $10^{10}$ or $10^{11} \mathrm{vp}$ of Ad5-Luc vector (6 mice/group) were immunized with $10^{10} \mathrm{vp}$ of Ad5HIV or Ad5/35-HIV vector. At the time of vaccination, anti-Ad5 neutralizing titers were measured in Ad5-Luc-treated mice. At 2 weeks after vaccination, the HIV-specific responses were detected by an HIV-specific tetramer assay. ${ }^{*}$ Mean values are significantly different between the groups. 
pre-existing immunity against the parent Ads (Table 2 and Figure 4). The exchange of other genes, including hexon and penton, may further reduce the inhibition of pre-existing anti-Ad5 immunity. (2) We also explored the immune responses by using the same vector for prime/boost. When the mice were immunized i.m. with $10^{10} \mathrm{vp}$ of Ad5/35-HIV 1-3 times at 4-week intervals, the HIV-specific cell-mediated immune responses were detected by the tetramer assay. An increased response was observed after the second immunization but not after the third immunization (data not shown). High anti-Ad5/35 neutralizing $\mathrm{Ab}$ after second immunization may block the Ad5/35-HIV infection (Figure 4). These results are in agreement with the data from our study on monkeys (Figure $3 b$ and $c$ ) and with that from studies by other groups, ${ }^{33,43}$ and other virus such as MVA vector may be applicable for further boost if high immune responses are required. The DNA-HIV vaccine prime/Ad5/35-HIV vector boost regimen greatly increased HIV-specific cell-mediated immune responses (Table 1 and Figure $3 b$ and $c$ ), but not the humoral immune response (Figure 2c). This suggests that DNA vaccination enhances adenoviral recombinant-induced cell-mediated immunity rather than humoral immunity, as described by other groups. ${ }^{4,38}$ Furthermore, DNA vaccine priming can reduce the humoral response to the adenoviral antigens and can counterbalance the impaired B-cell response to the antigen expressed by the adenoviral recombinant in mice that are preimmune to Ad. ${ }^{46}$ This DNA prime/Ad5/35 boost regimen might be highly suitable for use in humans with previous exposure to the Ad5 virus. (3) To examine the immunogenicity and protective immunity of the Ad5/35 vector, we used HIV-1 IIIB in this study, because the strain has been well studied and we can compare our data from the new vector with that from other studies. For the clinical trail, we have constructed DNA vaccine and Ad5/5 vector expressing ENV and GAG of a Clade C HIV-1 isolate and similar results with HIV-1 IIIB strain were obtained in a mouse model (manuscript in preparation).

Considered together, using Ad5/35 vector, we developed an HIV vaccine with a higher immunogenicity and low pathogenicity. The Ad5/35-HIV vaccine induced strong HIV-specific immune responses in both $\mathrm{BALB} / \mathrm{c}$ mice and rhesus monkeys. Priming with a DNA-HIV vaccine followed by Ad5/35-HIV boosting yielded protection against viral infection in mice. The Ad5/35 vector may be a promising vaccine for human clinical trial.

\section{Materials and methods}

\section{Recombinant vectors}

E1,E3-deletion, replication-defective recombinant viruses were constructed with an Ad generation kit (Avior Therapeutics Inc., Seattle, WA, USA). ${ }^{21}$ Briefly, a $5.2 \mathrm{k}$ bp SalI/PstI fragment containing CAG promoter-HIV IIIB rev/env gp160-polyA was isolated from pCAGrev/ env. $^{28}$ A shuttle plasmid (pLHSP) containing Ad5 positions 22-342, Ad5 3523-5790, Escherichia coli ori, and ampicillin-resistant gene was obtained from Avior Therapeutics Inc. (Seattle, WA, USA). The $5.2 \mathrm{k} \mathrm{bp}$ blunted fragment was subcloned into blunted EcoRI site of pLHSP plasmid vector to generate pLHSP-HIV shuttle plasmid. The pLHSP-HIV shuttle plasmid was linearized with PacI and transfected with E1,E3-deletion, chimeric Ad5 or 5/35 genome to human embryonic kidney (HEK293) cells using calcium precipitation method to generate recombinant virus, Ad5-HIV and Ad5/35-HIV, respectively. The recombinant virus (Ad5/35-HIV, Ad5HIV) was propagated in HEK293 cells and purified by two repetitions of the $\mathrm{CsCl}$ methods described elsewhere. ${ }^{47}$ The total concentration of virions in each preparation was calculated from the optical density at $260 \mathrm{~nm}\left(\mathrm{OD}_{260}\right)$, using the formula $1 \mathrm{OD}_{260}=1 \times 10^{12} \mathrm{vp} /$ $\mathrm{ml}$. The $\mathrm{HIV}_{\mathrm{BH}}$ gp160-expressing replication-competent vaccinia virus (WR strain, vPE16; $\mathrm{HIV}_{\mathrm{BH}}$ gp160 has 97.32\% amino-acid homology with $\mathrm{HIV}_{\text {IIIB }}$ gp160) was obtained from the AIDS Research and Reagent Program, National Institutes of Health, Rockville, MD, USA (Cat. No. 362). The vPE16 vectors were propagated in CV1 cells. The Ad5-Luc and Ad5/35-Luc vectors expressing luciferase coding gene were described previously. ${ }^{26}$ The DNA-HIV vaccine (pCAGrev/env) containing $\mathrm{HIV}_{\text {IIIB }}$ rev and $e n v$ genes has been previously reported. ${ }^{28}$

\section{Biodistribution of Ad5 and Ad5/35 vectors in vivo}

The experiment was performed as previously described. ${ }^{48,49}$ In brief, the Ad5-Luc or Ad5/35-Luc vectors $\left(10^{11} \mathrm{vp} / \mathrm{mouse}\right)$ were injected i.m. into BALB/c mice. On days 3 and 10, the mice were anesthetized with a $2 \%$ isofluorane/air mixture, and a single dose of $150 \mathrm{mg} / \mathrm{kg}$ luciferin in normal saline was administered intraperitoneally. The CCD images were obtained using a cooled in vivo imaging system (IVIS) CCD camera (Xenogen, Alameda, CA, USA) and analyzed. To study the viral biodistribution in primates, two rhesus monkeys (2 years old, male) were administered i.m. $10^{11} \mathrm{vp}$ of Ad5-Luc or Ad5/35-Luc. At 3 days after administration, the luciferase activity was detected in the monkey organs (brain, liver, kidney, spleen, heart, and lung) using the Luciferase Assay Systems (Promega, Madison, WI, USA). Serum GOT and serum GPT were measured in mouse or monkey sera at the Kitayama-Rabesu Institute (Ina, Nagano, Japan). The concentration of serum IFN- $\gamma$ and IL-6 was measured using the IFN- $\gamma$ and IL-6 ELISA kits (Biosource, Camarillo, CA, USA), respectively, according to the manufacturer's protocol.

\section{Animal immunization}

Female BALB/c mice (8-week-old; $\mathrm{H}-2 \mathrm{D}^{\mathrm{d}}$ ) were purchased from Japan SLC Inc., Shizuoka, Shizuoka-ken, Japan. The mice were immunized with an i.m. injection of $100 \mu \mathrm{g}$ of pCAGrev/env or pCAGempty plasmid DNA in phosphate-buffered saline (PBS) at 0,1 , and 2 weeks and were boosted with $10^{10} \mathrm{vp}$ of Ad5/35-HIV or Ad5/35-Luc vector at week 3 . For the time-course study, the mice were administered a single i.m. injection of $10^{10} \mathrm{vp}$ of Ad5/35-HIV vector per mouse. To study the effect of pre-existing antiviral immunity on vaccination, the mice were injected i.m. with $10^{10}$ or $10^{11} \mathrm{vp}$ of Ad5Luc vector and then immunized 8 weeks later with $10^{10} \mathrm{vp}$ of Ad5-HIV vector or Ad5/35-HIV vector. For rhesus monkey immunization, $10^{11} \mathrm{vp}$ of Ad5/35-HIV vector was injected i.m. into two rhesus monkeys (2 years old, male) at weeks 0 and 8 . 
Intracellular cytokine staining assay

IFN- $\gamma$-secreting $\mathrm{CD}^{+} \mathrm{T}$ cells were detected by the protocol recommended by the manufacturer (Cytofix/ CytoPerm Plus kit, PharMingen, San Diego, CA, USA). In brief, lymphocytes were isolated from the mouse spleen. A single cell suspension was incubated with $10 \mu \mathrm{g} / \mathrm{ml}$ of the HIV V3 peptide (NNTRKRIQRGP GRAFVTIGKIGN) for $24 \mathrm{~h}$ at $37^{\circ} \mathrm{C}$. At $2 \mathrm{~h}$ before the end of incubation, $1 \mu \mathrm{g} / \mathrm{ml}$ of GolgiPlug was added. The cells were washed with staining buffer ( $3 \%$ fetal calf serum (FCS), $0.1 \%$ sodium azide $\left(\mathrm{NaN}_{3}\right)$ in PBS), blocked with $4 \%$ normal mouse sera, and stained with phycoerythrin (PE)-conjugated anti-mouse CD8 Ab (Ly-2, PharMingen). The cells were then suspended in $250 \mu \mathrm{l}$ of Cytofix/Cytoperm solution at $4^{\circ} \mathrm{C}$ for $20 \mathrm{~min}$, washed with Perm/Wash solution, and stained with anti-mouse IFN- $\gamma \mathrm{Ab}$ conjugated with fluorescein isothiocyanate (FITC) (PharMingen) at $4^{\circ} \mathrm{C}$ for $30 \mathrm{~min}$, followed by flow cytometric analysis.

\section{Tetramer assay}

The tetramer assay used a PE-conjugated H-2D $/ \mathrm{p} 18$ tetramer (RGPGRAFVTI), as previously described. ${ }^{28}$ In brief, splenocytes were isolated from mice and incubated for $30 \mathrm{~min}$ at $4{ }^{\circ} \mathrm{C}$ with $4 \%$ normal mouse serum in PBS. Cells were stained with FITC-conjugated anti-mouse CD8 Ab (Ly-2, PharMingen) for $30 \mathrm{~min}$ at $4^{\circ} \mathrm{C}$. After washing twice with the staining buffer (3\% FCS, $0.1 \%$ $\mathrm{NaN}_{3}$ in PBS), the cells were incubated with the tetramer reagent for $15 \mathrm{~min}$ at $37^{\circ} \mathrm{C}$, followed by flow cytometric analysis (Becton Dickinson).

\section{Recombinant vaccinia virus used for the challenge study}

Using vPE16 vaccinia virus, the virus challenge experiment was performed as described previously. ${ }^{28}$ Vaccinated female mice were intraperitoneally challenged with $10^{8}$ PFU of vaccinia virus vPE16 at 2 or 7 weeks after the final immunization. At 6 days after challenge, the mice were killed, their ovaries were sonicated, and the vPE16 titer was determined by serial 10-fold dilution on a plate of CV1 cells. Infected cells were detected by staining with crystal violet and plaques were counted at each dilution.

\section{Detection of HIV-1-specific Ab}

The HIV-1-specific $\mathrm{Ab}$ was detected by the Western blotting method and the enzyme-linked immunosorbent assay (ELISA). By Western blotting method, the HIV envelope glycoprotein gp160-coated membrane from the New Lav Blot 1 kit (Bio-Rad, Marnes-la-Coquette, France) was incubated with a 100-fold dilution of mouse serum followed by an affinity-purified horseradish peroxidase (HRP)-labeled anti-mouse immunoglobulin (ICN Pharmaceuticals Inc., OH, USA). HIV gp160 protein was detected using the ECL Plus Western Blotting Detection System (Amersham Pharmacia Biotech).

ELISA was performed as described elsewhere. ${ }^{10}$ To summarize, 96-well microtiter plates were coated with $10 \mu \mathrm{g} / \mathrm{ml}$ of $\mathrm{HIV}_{\text {IIIв }} \mathrm{gp} 120$ protein (donated by AIDS Research and Reference Reagent Program, National Institutes of Health) and incubated overnight at $4^{\circ} \mathrm{C}$. The wells were blocked with PBS containing $1 \%$ bovine serum albumin (BSA) for $2 \mathrm{~h}$ at room temperature. They were then treated with $100 \mu$ l of serially diluted antisera and incubated for an additional $2 \mathrm{~h}$ at $37^{\circ} \mathrm{C}$. The bound immunoglobulin was quantified using an affinitypurified HRP-labeled anti-mouse $\mathrm{Ab}$ or anti-monkey $\mathrm{Ab}$ (both from Sigma). The mean $\mathrm{Ab}$ titer was expressed as the reciprocal of the serial serum dilution that exceeded the assay background by 2 s.d.

The HIV-specific neutralizing titer of immune mice or monkeys was also measured. The serially diluted antisera were incubated with 200-300 blue spot-forming units (BFU) of $\mathrm{HIV}-1_{\mathrm{LAI}}$ at $37^{\circ} \mathrm{C}$ for $1 \mathrm{~h}$. The mixture was incubated with confluent MAGIC5 cells (from Dr Tatsumi, National Institute of Infectious Diseases, Tokyo, Japan), ${ }^{50,51}$ Dulbecco's modified Eagle's medium (DMEM) with $10 \%$ FCS and $0.2 \mathrm{mg} / \mathrm{ml}$ of G418 in a 96-well plate at $37^{\circ} \mathrm{C}$ for 2 days. The cells were fixed with fixing solution ( $1 \%$ formaldehyde, $0.2 \%$ glutaraldehyde in PBS) for $5 \mathrm{~min}$ and stained with staining solution (4 mM potassium ferrocyanide, $4 \mathrm{~mm}$ potassium ferricyanide, $2 \mathrm{mM}$ magnesium chloride, $0.4 \mathrm{mg} / \mathrm{ml} \mathrm{X}$-gal in PBS) at $37^{\circ} \mathrm{C}$ for $18-24 \mathrm{~h}$. The staining was stopped by removing the staining solution and the cells were washed twice with PBS. The blue spot in each well was counted after the staining, and the neutralizing titer was calculated as $(1-(\%$ infection $/ \%$ infection of control wells $) \times 100$. The $50 \%$ neutralization dose $\left(\mathrm{ND}_{50}\right)$ is defined as the concentration of the $\mathrm{Ab}$ that reduced the number of infected cells by $50 \%$. The detecting limitation of the assay was $100 \mathrm{ND}_{50} / \mathrm{ml}$.

\section{ELISPOT assay}

The frequency of HIV-specific IFN- $\gamma$-secreting cells in monkeys was determined using an ELISPOT assay kit (U-Cytech, Utrech, The Netherlands) according to the manufacturer's manual. In brief, $2 \times 10^{5}$ monkey PBMCs were stimulated in triplicate wells with $1 \mu \mathrm{g} / \mathrm{ml}$ of the $\mathrm{HIV}_{\text {IIIв }}$ gp120 protein for $16 \mathrm{~h}$ at $37^{\circ} \mathrm{C}$. Nonstimulated cells were used to assess the background. The cells were transferred to an anti-IFN- $\gamma$ Ab-coated 96-well plate and incubated for $5 \mathrm{~h}$ at $37^{\circ} \mathrm{C}$. The cells were removed and $200 \mu \mathrm{l} /$ well of ice-cold deionized water was added to lyse the remaining PBMCs. Subsequently, the plate was washed with PBS containing 0.05\% Tween 20 (PBS-T) and incubated with biotinylated anti-IFN- $\gamma \mathrm{Ab}$ for $1 \mathrm{~h}$ at $37^{\circ} \mathrm{C}$. After 10 washings with PBS-T, $50 \mu \mathrm{l}$ of gold-labeled anti-biotin $\mathrm{Ab}$ was added and incubated for $1 \mathrm{~h}$ at $37^{\circ} \mathrm{C}$. The plate was washed 10 times with PBS-T, and $30 \mathrm{ml}$ of activator solutions was added. The plate was incubated in the dark for $30 \mathrm{~min}$ at room temperature to develop spot formations. After $30 \mathrm{~min}$ incubation, the plate was washed with deionized water and air-dried; spots were counted by a computer-assisted video image analysis. The results were expressed as spot-forming cells (SFC) per million cells.

\section{Ad-specific neutralizing assay}

Ad5-Luc or Ad5/35-Luc vector $\left(10^{7} \mathrm{vp}\right)$ was incubated with an equal volume of serially diluted normal human sera (anti-Ad5 neutralizing titer $<1: 4$ ), human antisera (anti-Ad5 neutralizing titer $=1: 64$ ), or monkey sera (at weeks $0,2,8$, and 12 after immunization with Ad5/35HIV vector) at $37^{\circ} \mathrm{C}$ for $2 \mathrm{~h}$. The mixture was incubated with confluent Vero cells in a 96 -well plate at $37^{\circ} \mathrm{C}$ for an additional $48 \mathrm{~h}$. The luciferase activity was detected by Luciferase Assay Systems (Promega, Madison, WI, USA). 
The neutralizing titer was calculated with limited serum dilution when the luciferase activity in the Ad-infected cells was equal with the background.

\section{Data analysis}

All values were expressed as means \pm standard error (s.e.). Statistical analysis of the experimental data and controls was conducted with one-way factorial analysis of variance. Significance was defined at $P<0.05$ in the statistical analysis.

\section{Acknowledgements}

We are grateful to M Kawano, T Takeishi, and T Matsuda for their technical assistance and A De La Fuente for her secretarial assistance. This work was partially supported by a Grant-in-Aid from the Ministry of Education, Science, Sports, and Culture of Japan, and the grant for 2005 Strategic Research Project (No. K17018) of Yokohama City University, Japan.

\section{References}

1 Bukawa $\mathrm{H}$ et al. Neutralization of HIV-1 by secretory $\operatorname{IgA}$ induced by oral immunization with a new macromolecular multicomponent peptide vaccine candidate. Nat Med 1995; 1: 681-685.

2 Barouch DH et al. Control of viremia and prevention of clinical AIDS in rhesus monkeys by cytokine-augmented DNA vaccination. Science 2000; 290: 486-492.

3 Robinson HL et al. Neutralizing antibody-independent containment of immunodeficiency virus challenges by DNA priming and recombinant pox virus booster immunizations. Nat Med 1999; 5: 526-534.

4 Shiver JW et al. Replication-incompetent adenoviral vaccine vector elicits effective anti-immunodeficiency-virus immunity. Nature 2002; 415: 331-335.

5 Patterson LJ et al. Protection against mucosal simian immunodeficiency virus SIV(mac251) challenge by using replicating adenovirus-SIV multigene vaccine priming and subunit boosting. J Virol 2004; 78: 2212-2221.

6 Schnell MJ et al. Recombinant rabies virus as potential live-viral vaccines for HIV-1. Proc Natl Acad Sci USA 2000; 97: 3544-3549.

7 Mandl CW et al. In vitro-synthesized infectious RNA as an attenuated live vaccine in a flavivirus model. Nat Med 1998; 4: 1438-1440.

8 Matano T et al. Cytotoxic T lymphocyte-based control of simian immunodeficiency virus replication in a preclinical AIDS vaccine trial. J Exp Med 2004; 199: 1709-1718.

9 Caley IJ et al. Venezuelan equine encephalitis virus vectors expressing HIV-1 proteins: vector design strategies for improved vaccine efficacy. Vaccine 1999; 17: 3124-3135.

10 Xin KQ et al. Oral administration of recombinant adenoassociated virus elicits human immunodeficiency virus-specific immune responses. Hum Gene Ther 2002; 13: 1571-1581.

11 Xin KQ et al. A novel recombinant adeno-associated virus vaccine induces a long-term humoral immune response to human immunodeficiency virus. Hum Gene Ther 2001; 12: 1047-1061.

12 Aldovini A, Young RA. Humoral and cell-mediated immune responses to live recombinant BCG-HIV vaccines. Nature 1991; 351: $479-482$.

13 Someya $\mathrm{K}$ et al. Vaccination of rhesus macaques with recombinant Mycobacterium bovis bacillus Calmette-Guerin Env V3 elicits neutralizing antibody-mediated protection against simianhuman immunodeficiency virus with a homologous but not a heterologous V3 motif. J Virol 2005; 79: 1452-1462.
14 Xin KQ et al. Immunogenicity and protective efficacy of orally administered recombinant Lactococcus lactis expressing surfacebound HIV Env. Blood 2003; 102: 223-228.

15 Sullivan NJ et al. Development of a preventive vaccine for Ebola virus infection in primates. Nature 2000; 408: 605-609.

16 Amara RR et al. Different patterns of immune responses but similar control of a simian-human immunodeficiency virus 89.6P mucosal challenge by modified vaccinia virus Ankara (MVA) and DNA/MVA vaccines. J Virol 2002; 76: 7625-7631.

17 Vogels $\mathrm{R}$ et al. Replication-deficient human adenovirus type 35 vectors for gene transfer and vaccination: efficient human cell infection and bypass of preexisting adenovirus immunity. J Virol 2003; 77: 8263-8271.

18 Havenga MJ et al. Exploiting the natural diversity in adenovirus tropism for therapy and prevention of disease. J Virol 2002; 76: 4612-4620.

19 Roelvink PW et al. The coxsackievirus-adenovirus receptor protein can function as a cellular attachment protein for adenovirus serotypes from subgroups A, C, D, E, and F. J Virol 1998; 72: 7909-7915.

20 Sakurai F, Mizuguchi H, Yamaguchi T, Hayakawa T. Characterization of in vitro and in vivo gene transfer properties of adenovirus serotype 35 vector. Mol Ther 2003; 8: 813-821.

21 Shayakhmetov DM, Papayannopoulou T, Stamatoyannopoulos G, Lieber A. Efficient gene transfer into human CD34(+) cells by a retargeted adenovirus vector. J Virol 2000; 74: 2567-2583.

22 Adams JY et al. Visualization of advanced human prostate cancer lesions in living mice by a targeted gene transfer vector and optical imaging. Nat Med 2002; 8: 891-897.

23 Thomas CE, Ehrhardt A, Kay MA. Progress and problems with the use of viral vectors for gene therapy. Nat Rev Genet 2003; 4: 346-358.

24 Gao W, Robbins PD, Gambotto A. Human adenovirus type 35: nucleotide sequence and vector development. Gene Therapy 2003; 10: 1941-1949.

25 Gaggar A, Shayakhmetov DM, Lieber A. CD46 is a cellular receptor for group B adenoviruses. Nat Med 2003; 9: 1408-1412.

26 Mizuguchi $\mathrm{H}$, Hayakawa T. Adenovirus vectors containing chimeric type 5 and type 35 fiber proteins exhibit altered and expanded tropism and increase the size limit of foreign genes. Gene 2002; 285: 69-77.

27 Seshidhar Reddy P et al. Development of adenovirus serotype 35 as a gene transfer vector. Virology 2003; 311: 384-393.

28 Jounai $\mathrm{N}$ et al. Contribution of the rev gene to the immunogenicity of DNA vaccines targeting the envelope glycoprotein of HIV. J Gene Med 2003; 5: 609-617.

29 Maino VC, Picker LJ. Identification of functional subsets by flow cytometry: intracellular detection of cytokine expression. Cytometry 1998; 34: 207-215.

30 Letvin NL et al. Heterologous envelope immunogens contribute to AIDS vaccine protection in rhesus monkeys. J Virol 2004; 78: 7490-7497.

31 Barouch DH et al. Reduction of simian-human immunodeficiency virus $89.6 \mathrm{P}$ viremia in rhesus monkeys by recombinant modified vaccinia virus Ankara vaccination. J Virol 2001; 75: 5151-5158.

32 Altman JD et al. Phenotypic analysis of antigen-specific $\mathrm{T}$ lymphocytes. Science 1996; 274: 94-96.

33 Sullivan NJ et al. Accelerated vaccination for Ebola virus haemorrhagic fever in non-human primates. Nature 2003; 424: 681-684.

34 Wadell G. Molecular epidemiology of human adenoviruses. Curr Top Microbiol Immunol 1984; 110: 191-220.

35 De Jong JC et al. Adenoviruses from human immunodeficiency virus-infected individuals, including two strains that represent new candidate serotypes Ad50 and Ad51 of species B1 and D, respectively. J Clin Microbiol 1999; 37: 3940-3945. 
36 Kostense $\mathrm{S}$ et al. Adenovirus types 5 and 35 seroprevalence in AIDS risk groups supports type 35 as a vaccine vector. Aids 2004; 18: 1213-1216.

37 Barouch DH et al. Immunogenicity of recombinant adenovirus serotype 35 vaccine in the presence of pre-existing anti-Ad5 immunity. J Immunol 2004; 172: 6290-6297.

38 Casimiro DR et al. Comparative immunogenicity in rhesus monkeys of DNA plasmid, recombinant vaccinia virus, and replication-defective adenovirus vectors expressing a human immunodeficiency virus type 1 gag gene. J Virol 2003; 77: 6305-6313.

39 Morral $\mathrm{N}$ et al. Administration of helper-dependent adenoviral vectors and sequential delivery of different vector serotype for long-term liver-directed gene transfer in baboons. Proc Natl Acad Sci USA 1999; 96: 12816-12821.

40 Hofmann $C$ et al. Ovine adenovirus vectors overcome preexisting humoral immunity against human adenoviruses in vivo. J Virol 1999; 73: 6930-6936.

41 Farina SF et al. Replication-defective vector based on a chimpanzee adenovirus. J Virol 2001; 75: 11603-11613.

42 Moffatt S, Hays J, HogenEsch H, Mittal SK. Circumvention of vector-specific neutralizing antibody response by alternating use of human and non-human adenoviruses: implications in gene therapy. Virology 2000; 272: 159-167.

43 Pinto AR et al. Induction of CD8+ T cells to an HIV-1 antigen through a prime boost regimen with heterologous E1-deleted adenoviral vaccine carriers. J Immunol 2003; 171: 6774-6779.
44 Fitzgerald JC et al. A simian replication-defective adenoviral recombinant vaccine to HIV-1 gag. J Immunol 2003; 170: 1416-1422.

45 Lemiale $\mathrm{F}$ et al. Enhanced mucosal immunoglobulin A response of intranasal adenoviral vector human immunodeficiency virus vaccine and localization in the central nervous system. J Virol 2003; 77: 10078-10087.

46 Xiang ZQ, Pasquini S, Ertl HC. Induction of genital immunity by DNA priming and intranasal booster immunization with a replication-defective adenoviral recombinant. J Immunol 1999; 162: 6716-6723.

47 Lieber A, He CY, Kirillova I, Kay MA. Recombinant adenoviruses with large deletions generated by Cre-mediated excision exhibit different biological properties compared with firstgeneration vectors in vitro and in vivo. J Virol 1996; 70: 8944-8960.

48 Bhaumik S, Gambhir SS. Optical imaging of Renilla luciferase reporter gene expression in living mice. Proc Natl Acad Sci USA 2002; 99: 377-382.

49 Lipshutz GS et al. In utero delivery of adeno-associated viral vectors: intraperitoneal gene transfer produces long-term expression. Mol Ther 2001; 3: 284-292.

50 Mochizuki $\mathrm{N}$ et al. An infectious DNA clone of HIV type 1 subtype C. AIDS Res Hum Retroviruses 1999; 15: 1321-1324.

51 Mukai T et al. Construction and characterization of an infectious molecular clone derived from the CRF01_AE primary isolate of HIV type 1. AIDS Res Hum Retroviruses 2002; 18: 585-589. 